\title{
Long term benzodiazepine use for insomnia in patients over the age of 60: discordance of patient and physician perceptions Leevin Mah ${ }^{1}$ and Ross EG Upshur *2
}

\author{
Address: ${ }^{1}$ Family Medicine Residency Program, Department of Family and Community Medicine Sunnybrook and Women's College Health \\ Sciences Centre, University of Toronto, Toronto, Ontario, Canada and ${ }^{2}$ Primary Care Research Unit, Department of Family and Community \\ Medicine Sunnybrook and Women's College Health Sciences Centre, University of Toronto 2075 Bayview Avenue, Toronto, Ontario, M4N 3M5, \\ Canada \\ E-mail: Leevin Mah - leevin.mah@utoronto.ca; Ross EG Upshur* - rupshur@idirect.com \\ ${ }^{*}$ Corresponding author
}

Published: 8 May 2002

BMC Family Practice 2002, 3:9
Received: 20 March 2002

Accepted: 8 May 2002

This article is available from: http://www.biomedcentral.com/I47I-2296/3/9

(C) 2002 Mah and Upshur; licensee BioMed Central Ltd. Verbatim copying and redistribution of this article are permitted in any medium for any purpose, provided this notice is preserved along with the article's original URL.

\begin{abstract}
Background: The aim of this study was to determine and compare patients' and physicians' perceptions of benefits and risks of long term benzodiazepine use for insomnia in the elderly.

Methods: A cross-sectional study (written survey) was conducted in an academic primary care group practice in Toronto, Canada. The participants were 93 patients over 60 years of age using a benzodiazepine for insomnia and 25 physicians comprising sleep specialists, family physicians, and family medicine residents. The main outcome measure was perception of benefit and risk scores calculated from the mean of responses (on a Likert scale of $I$ to 5 ) to various items on the survey.

Results: The mean perception of benefit score was significantly higher in patients than physicians ( 3.85 vs. $2.84, \mathrm{p}<0.00 \mathrm{I}, 95 \% \mathrm{Cl} 0.69, \mathrm{I} .32$ ). The mean perception of risk score was significantly lower in patients than physicians $(2.21$ vs. $3.63, \mathrm{p}<0.00 \mathrm{I}, 95 \% \mathrm{Cl}$ I.07, I.77).

Conclusions: There is a significant discordance between older patients and their physicians regarding the perceptions of benefits and risks of using benzodiazepines for insomnia on a long term basis. The challenge is to openly discuss these perceptions in the context of the available evidence to make collaborative and informed decisions.
\end{abstract}

\section{Background}

Many older people suffer from insomnia and are commonly prescribed benzodiazepines for symptomatic management. Among adults aged 65 and older who live in the community, up to $42 \%$ reported difficulty falling asleep or staying asleep [1]. The prevalence of benzodiazepine prescriptions in older people is also high: $22.5 \%$ of people aged 65 and older in Ontario, Canada reported use of benzodiazepines [2]. Furthermore, older people are more likely to continue using these medications for extended periods once they are prescribed them [3].

The research available on the benefits and risks of benzodiazepine use for insomnia in older people is surprisingly deficient for the magnitude of this issue. Notably, there are no prospective studies that have looked at the efficacy of benzodiazepine use for insomnia for a duration of greater than one month [4]. Over the short term, metaanalyses show a mild to moderate treatment effect in 
quantitative and subjective measures of sleep [5,6]. However, in practice, patients may be taking these medications for over a year or more.

There are concerns about prescribing benzodiazepines because of potential adverse effects. However, there are no studies that clearly demonstrate adverse effects due to long term use of short acting benzodiazepines in older populations for insomnia. The evidence that does exist is limited by small sample sizes, short durations, or not addressing the population of interest, namely communitydwelling patients aged sixty and over. Benzodiazepines have been associated with increased risk in hip fractures [7-9], motor vehicle crashes [10], and cognitive impairment [11]. The significance of these associations is unclear. While some studies have shown increased risks in long acting benzodiazepines, higher doses, or longer durations of therapy, others have shown none. Addiction and dose escalation are also widespread concerns, despite no long term studies to validate them. Similarly, the adverse effects of benzodiazipine withdrawl in older patients using benzodiazepines for insomnia are unclear. What is clear is that there is no consensus on the overall risks of benzodiazepine use for insomnia in older persons as the evidence to date is either inconclusive, conflicting, or anecdotal.

With the paucity of clinically relevant and long term research in older populations, physicians lack evidencebased guidelines on how to advise long term users of benzodiazepines. Yet the common dilemma arises when an older patient requests the renewal of the benzodiazepine from a physician who has reservations due to the perceived risks. This study sought to quantify this potential discordance between patients and physicians with regard to long term benzodiazepine use for insomnia.

\section{Methods}

The subjects in the patient group were recruited from the practices of thirteen family physicians in an academic family practice in Toronto, Canada. By reviewing telephone records, patients who renewed a prescription for a benzodiazepine from January to June 2001 were identified as potential subjects. Patients 60 years and older and receiving benzodiazepine prescriptions for greater than one year duration were included. The list of subjects was then reviewed by their respective family doctors. The physician could veto sending a survey to a patient for various reasons such as undue psychological distress of receiving the survey, inability to complete the form due to physical or cognitive impairment, or because the patient was not using the medication for insomnia.

The physician group sample consisted of all staff and resident physicians at the academic family practice. It also in- cluded the sleep specialists in three academic teaching hospitals affiliated with the University of Toronto.

The survey was pretested among a small sample of physicans and patients $(n=6)$ to ensure clarity of wording. Surveys were mailed out to all eligible patient and physician subjects. A follow-up mailing was conducted one month after the initial one to those who did not respond.

\section{Survey design}

Seven items including one for overall opinion were used to assess the benefits of benzodiazepine use. They were based on criteria described by Dement which included sleep latency, night time awakenings, total sleep time, and feeling of being well-rested on awakening [4]. Improved daytime function and overall well-being have also been noted to be important clinical outcomes and were included [6]. Regarding the risks, subjects were asked to respond to nine questions on potential adverse effects of benzodiazepine use that have been identified in the literature [5]. These included daytime drowsiness, confusion, cognitive impairment, concentration problems, dependence, falls, hip fractures, motor vehicle crashes, and overall assessment of risk.

The patients and physicians were asked the same items regarding their perceptions of specific benefits and risks of benzodiazepine use. While the patients responded based on their personal experiences, the physicians provided their perceptions for a hypothetical older person using a short-acting benzodiazepine on a long term basis. The survey employed a Likert scale ranging from one to five, which correlated to strongly disagreeing to strongly agreeing to the various statements. Additional information requested from patient subjects included the name of sleeping medication, frequency and duration of use, other methods used to help sleep, and comorbid conditions.

\section{Data analysis}

The main outcome measures were the mean benefit and risk scores. A benefit score was calculated for each subject by taking the mean of all the responses from the questions on perceived benefits. Likewise, a risk score was calculated by taking the mean of responses from the questions on perceived risk. The scores potentially ranged from a minimum of 1 to a maximum of 5 as per the Likert scale. A Ttest for independent samples was conducted to assess statistical significance between the scores for patients and physicians.

\section{Ethics}

The research ethics board of Sunnybrook and Women's College Health Sciences Centre in Toronto, Canada approved the study question and protocol. 


\section{Results}

Two hundred and forty-two potential patient subjects were initially identified through review of telephone renewal records (Figure 1). Of these subjects, 44 were excluded by the family physician for various reasons, most commonly for undue anxiety (21) or no longer with practice due to death or moving (11). One hundred and ninety-eight surveys were mailed out. Five surveys did not reach their destinations. After two separate mailings, 131 responses were received from patients. Of these, 23 said they were not currently taking a medication for insomnia. Another 14 were not using a benzodiazepine, and three did not specify their medication. Ninety-three returned surveys from current benzodiazepine users were analyzed. The response rate was $61 \%$ for patient subjects who met the inclusion criteria.

Thirty-nine potential physician subjects were identified (11 sleep specialists, 12 family doctors, 16 family medicine residents). Twenty-five surveys were returned. One survey did not reach its destination. The response rate was $66 \%$ for physician subjects.

Table 1 shows demographic characteristics of patients who responded, did not respond, and those who were initially excluded by their family doctors. There was no statistically significant difference between the groups. The average age of responders was 77 years old. The percentage of female responders was $67 \%$.

The types of benzodiazepines used by the patient subjects varied (Table 2). Eleven percent were using a long acting benzodiazepine such as diazepam, clonazepam or flurazepam. Eighty-nine percent were using a short acting benzodiazepine, the three most common being lorazepam, temazepam, and oxazepam.
Subjects identified

242<smiles>C[14CH3]</smiles>

44 Excluded by their MD's

198

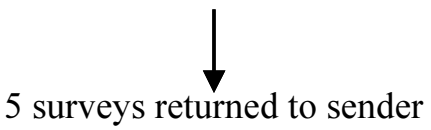

193<smiles>C[14CH3]</smiles>

Responded to Survey

133

\section{Using a}

Medication

110
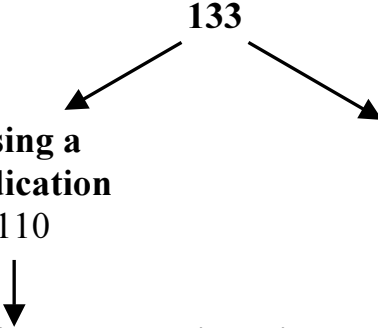

\section{3 using a benzodiazepine}

$14 *$ not using a benzodiazepine

$3 *$ did not specify

$$
\text { Return rate }=\frac{93}{193-40^{*}}=61 \%
$$

*excluded

\section{Figure I}

Recruitment and Response Rate

Table I: Demographic Characteristics

\begin{tabular}{|c|c|c|c|}
\hline Characteristics & Respondents $(n=93)$ & Non-Respondents $(n=61)$ & $\begin{array}{l}\text { Not authorized to receive survey } \\
\qquad(n=43)\end{array}$ \\
\hline \multicolumn{4}{|l|}{ Age Group (\%) } \\
\hline $60-69$ & 20 & 18 & 16 \\
\hline 70-79 & 32 & 38 & 30 \\
\hline $80-89$ & 42 & 33 & 53 \\
\hline $90-99$ & 5 & 11 & 0 \\
\hline Mean Age (yrs) & 77 & 78 & 78 \\
\hline \multicolumn{4}{|l|}{ Gender (\%) } \\
\hline Male & 33 & 26 & 23 \\
\hline Female & 67 & 74 & 77 \\
\hline
\end{tabular}


Table 2: Benzodiazepines Used by Patient Subjects

\begin{tabular}{lc}
\hline \multicolumn{1}{c}{ Medication } & No. of Patients \\
\hline $\begin{array}{l}\text { Long Elimination Half Life ( I \%) } \\
\text { Diazepam } \\
\text { Clonazepam } \\
\text { Flurazepam }\end{array}$ & 5 \\
& 4 \\
Short Elimination Half Life (89\%) & $\mathrm{I}$ \\
Lorazepam & \\
Temazepam & \\
Oxazepam & \\
Triazolam & 60 \\
Lectopam & 15 \\
& 4 \\
\hline
\end{tabular}

Calculated mean benefit and risk scores for patient and physician groups are shown in Table 3 . The mean score for perceived benefit from benzodiazepines among patients (3.85) was higher than physicians subjects (2.84). The difference of 1.01 was statistically significant ( $p<0.001$ ) with a $95 \%$ CI of $0.69-1.32$. The mean score for perceived risk among patients (2.21) was lower than that for physicians (3.63). The difference of 1.43 was also significant ( $p$ $<0.001$ ) with a $95 \%$ CI of 1.07-1.77.

Table 3: Mean Benefit and Risk Scores - Patients vs. Physicians

\begin{tabular}{cccc}
\hline & $\begin{array}{c}\text { Patients } \\
(\mathbf{n}=93)\end{array}$ & $\begin{array}{c}\text { Physicians } \\
(\mathbf{n}=25)\end{array}$ & Difference \\
\hline $\begin{array}{c}\text { Mean Benefit } \\
\text { Score }\end{array}$ & 3.84 & 2.84 & $1.00 \begin{array}{c}(0.69-1.32) P \\
<0.001\end{array}$ \\
$\begin{array}{c}\text { Mean Risk } \\
\text { Score }\end{array}$ & 3.63 & 2.21 & $1.42 \begin{array}{c}(1.07-1.77) P \\
<0.001\end{array}$ \\
\hline
\end{tabular}

\section{Discussion}

To our knowledge, this study is the first to directly compare the perceived benefits and risks of benzodiazepine use for insomnia in patients and physicians. It showed a clear discordance in perceptions. Overall, the patients felt there was generally good benefit in using benzodiazepines on a long term basis for insomnia whereas the physicians felt the benefits were neutral. With respect to the risks of using benzodiazepines, the patients generally felt they were low whereas the physicians felt they were high.
Is this difference clinically relevant? We think this discordance is of clinical importance because it is likely not addressed explicitly in the patient encounter. Concordance is an important clinical issue in primary care. Recent research has demonstrated that misunderstandings between patients and physicians seem to be associated with patients' lack of participation and are often based on assumptions about medications that are not made explicit in the clinical encounter [12]. There is also compelling evidence of a lack of open discourse between patients and physicians concerning the benefits and risks of prescription medication. The resulting discordance can lead to non-adherence or potentially adverse consequences of taking a medication. In the area of long term benzodiazepine use for insomnia in the patients aged sixty and over, there is a large gap in perceptions that needs to be addressed. Recent work in shared decision making provides a promising avenue of research to redress these gaps [13].

Part of the reason for the discordance may be the perceptions in the medical community towards the risks of benzodiazepines. Due in part to the negative publicity and perceived high levels of risk, there has been a trend since the 1970s towards decreased use in benzodiazepines [2]. The increased risks attributed to benzodiazepines have often been associated with long acting benzodiazepines or higher doses. Thus, small doses of short acting hypnotics, most commonly prescribed in this particular family practice, are generally viewed as safer. Although short acting benzodiazepines are not without risks in older people, the fear engendered in using these medications for patients is not substantiated by the current evidence.

With perceived benefits from patients and no convincing evidence of adverse outcomes, it can be argued that a long term use of short acting benzodiazepines may have therapeutic value. Should an older individual who is distressed by insomnia, but consistently responds well to a low-dose short acting benzodiazepine be denied this medication? Like all medical decisions, the patient should be made aware of the benefits and risks in a patient-centred approach. For benzodiazepines, the potential risks include possible low level dependence and rebound insomnia on discontinuation. On the other hand, the potential risks of not treating persistent insomnia can also be significant [14]. They may include poor daytime functioning, decreased mood, and decreased sense of well being. Decisions about using benzodiazepines for insomnia can be complex, and many factors in the patient's context should be considered.

\section{Alternatives}

There is a growing amount of research available on alternatives to benzodiazepines for insomnia. Non-prescrip- 
tion sleep products [15] and newer short acting nonbenzodiazepine medications such as zopiclone (Imovane $^{\circledast}$ ) and zaleplon (Starnoc ${ }^{\circledR}$ ) are increasingly used by patients. Their role versus traditional benzodiazepines with respect to efficacy or harm has yet to be demonstrated [16]. Furthermore, cognitive behavioural therapy (CBT) $[17,18]$ shows promising results of long term sleep improvements, and would be a non-pharmacologic consideration where available.

\section{Limitations}

There are a number of limitations to this survey. First, the sample for this survey was non-random and taken from a single academic family practice. Although there is no apparent reason to believe that these patients would respond differently to others in the community at large, generalizability is a potential issue. Second, the possibility of response bias may come from potential difficulties for some patients to complete the survey due to decreased cognitive or physical ability. Additionally, there is a possibility that respondants believed that a less than favourable account of their medication could result in its withdrawal. Finally, the goal of the survey was to quantify whether a discrepancy in the perceptions between patients and physicians existed. The reasons for this phenomenon were not explored. Further research in the form of in-depth qualitative studies with patients and physicians is required to further understand the reasons for this discordance.

\section{Conclusions}

There is a significant discordance between patients aged sixty and over and their physicians regarding the perceptions of risks and benefits of using short acting benzodiazepines for insomnia on a long term basis. Patients feel that benzodiazepines are more effective than the physicians that prescribe them. Physicians have traditionally been taught that benzodiazepines are generally risky and perhaps dangerous in the patients aged sixty and over. This perception is not shared by the patients, nor supported by the literature for short acting benzodiazepines.

When approaching insomnia in patients aged sixty and over, ruling out underlying medical and psychological disorders, medication causes, and environmental factors contributing to insomnia continues to be essential. Patients should also be aware of proper sleep hygiene and non-pharmacologic methods to treat insomnia that demonstrate evidence of being efficacious. Regarding benzodiazepine use in the patients aged sixty and over for insomnia, the evidence for safe and effective long term use is lacking. Thus there is an increased need for patients and physicians to openly discuss their perceptions with the available evidence to make collaborative and informed decisions. Recent research in shared decision making be- tween physicians and patients points out promising directions for this possibility.

\section{Competing interests}

There are no competing interests to declare

\section{Authors' contributions}

LM was involved in the conception, design, data collection, data entry, data analysis and interpretation, and drafting of the article. REGU was involved in the conception, design, interpretation of data, and revisions of the article. Both contributors agree on the contents of the final manuscript.

\section{Acknowledgements}

LM was supported by a bursary from the Department of Family and Community Medicine, Sunnybrook and Women's College Health Sciences Centre. REGU is supported by a New Investigator Award from the Canadian Institutes of Health Research and a Research Scholarship from the Department of Family and Community Medicine, University of Toronto. The authors would like to thank Shari Gruman for her assistance in the preparation of the manuscript.

\section{References}

I. Foley DJ, Monjan A, Simonsick EM, Wallace RB, Blazer DG: Incidence and remission of insomnia among elderly adults: an epidemiologic study of 6,800 persons over three years. Sleep 1999, 22(Suppl 2):S366-372

2. Tu K, Mamdani MM, Hux JE, Tu JB: Progressive trends in the prevalence of benzodiazepine prescribing in older people in Ontario, Canada. J Am Geriatr Soc 200I, 49: I34I-I345

3. Ancoli-Israel S: Insomnia in the elderly: a review for the primary care practitioner. Sleep 2000, 23(Suppl I):S23-30

4. Grad RM: Benzodiazepines for insomnia in community-dwelling elderly: a review of benefit and risk. J Fam Pract 1995, 41:473-48।

5. Nowell PD, Mazumdar S, Buysse DJ, Dew MA, Reynolds CF 3rd, Kupfer DJ: Benzodiazepines and zolpidem for chronic insomnia: a meta-analysis of treatment efficacy. JAMA 1997, 278:2 I70-2177

6. Holbrook AM, Crowther R, Lotter A, Cheng C, King D: Meta-analysis of benzodiazepine use in the treatment of insomnia. CMAJ 2000, I 62:225-233

7. Ray WA, Griffin MR, Schaffner W, Baugh DK, Melton LJ 3rd: Psychotropic drug use and the risk of hip fracture. N Engl J Med I987, 31 6:363-369

8. Ray WA, Griffin MR, Downey W: Benzodiazepines of long and short elimination half-life and the risk of hip fracture. JAMA 1989, 262:3303-3307

9. Pierfitte C, Macouillard G, Thicoipe M, Chaslerie A, Pehourcq F, Aissou M, Martinez B, Lagnaoui R, Fourrier A, Begaud B, et al: Benzodiazepines and hip fractures in elderly people: case-control study. BMJ 200I, 322:704-708

10. Hemmelgarn B, Suissa S, Huang A, Boivin JF, Pinard G: Benzodiazepine use and the risk of motor vehicle crash in the elderly. JAMA 1997, 278:27-3।

II. Larson EB, Kukull WA, Buchner D, Reifler BV: Adverse drug reactions associated with global cognitive impairment in elderly persons. Ann Intern Med 1987, I07:169-173

12. Britten N, Stevenson FA, Barry CA, Barber N, Bradley CP: Misunderstandings in prescribing decisions in general practice: qualitative study. BMJ 2000, 320:484-488

13. Wensing M, Elwyn G, Edwards A, Vingerhoets E, Grol R: Deconstructing patient centred communication and uncovering shared decision making: an observational study. BMC Med InfORM Dec Mak :2. [http://www.biomedcentral.com//472-6947/2/2]

14. Balter $M B$, Uhlenhuth $E H$ : The beneficial and adverse effects of hypnotics. J Clin Psychiatry I991, 52(Suppl): I6-23

15. Sproule BA, Busto UE, Buckle C, Herrmann N, Bowles S: The use of non-prescription sleep products in the elderly. Int J Geriatr Psychiatry 1999, 14:851-857 
16. Mitler MM: Nonselective and selective benzodiazepine receptor agonists - where are we today? Sleep 2000, 23(Suppl I):S39-47

17. Edinger JD, Wohlgemuth WK, Radtke RA, Marsh GR, Quillian RE: Cognitive behavioral therapy for treatment of chronic primary insomnia: a randomized controlled trial. JAMA 200I, 285: $1856-1864$

18. Morin CM, Colecchi C, Stone J, Sood R, Brink D: Behavioral and pharmacological therapies for late-life insomnia: a randomized controlled trial. JAMA I999, 28 I:991-999

\section{Pre-publication history}

The pre-publication history for this paper can be accessed here:

http://www.biomedcentral.com/1471-2296/3/9/prepub

Publish with BioMed Central and every scientist can read your work free of charge

"BioMedcentral will be the most significant development for disseminating the results of biomedical research in our lifetime."

Paul Nurse, Director-General, Imperial Cancer Research Fund

Publish with BMC and your research papers will be:

- available free of charge to the entire biomedical community

- peer reviewed and published immediately upon acceptance

- cited in PubMed and archived on PubMed Central

- yours - you keep the copyright

Submit your manuscript here:

http://www.biomedcentral.com/manuscript/
BioMedcentral.com editorial@biomedcentral.com 